11. - Über einen functionentheoretischen Satz des Herrn. G. Mittag-Leffler, Monatsbericht der Königl. Akademie der Wissenschaften (1880) (Werke, pp. 189199).

12. J. Worpitzky, Untersuchungen ibber die Entwickelung der Monodronen und mongenen Functionen durch Kettenbriuche, Friedrichs Gymnasium und Realschule, Jahresbericht (Berlin) 1865.

UNIVERSITY OF ILLINOIS

\title{
ARBITRARY MAPPINGS
}

H. D. BLOCK AND BUCHANAN CARGAL

The results of this paper generalize some results of $\mathrm{H}$. Blumberg. ${ }^{1}$

Let $X$ be a set of elements and let $\mathfrak{N}$ be a collection of nonempty subsets of $X$. We assume that there is, in $\mathfrak{R}$, a countable subcollection: $N^{1}, N^{2}, \cdots, N^{n}, \cdots$ with the property that, for each $N$ of $\mathfrak{R}$ and each $x$ of $N$, there is an integer $k$, such that $x \in N^{k}$ and $N^{k} \subseteq N$. In the remainder of this paper the letter $N$ with a superscript will always denote a member of the set $N^{1}, N^{2}, \cdots, N^{n}, \cdots$. The letter $N$ with or without subscripts will always denote a member of $\mathfrak{N}$, and the letter $x$ will denote an element of $X$. The symbol $N(x)$ denotes a set $N$ which contains $x$.

Let the statements of the preceding paragraph be repeated, replacing $X$ by $Y, \mathfrak{R}$ by $\mathfrak{M}, N$ by $M, x$ by $y$.

Let a correspondence, $f$, be given which to each $x$ assigns a nonempty subset (denoted by $f(x)$ ) of $Y$. If $V \subseteq Y$, then $f^{-1}(V)$ denotes the set of all $x$ such that $f(x) \cdot V \neq 0$.

Definitions. Let $S$ be a subset of $X$. Then $S$ is nowhere dense if, for each $N$, there is an $N_{1} \subseteq N$ with $N_{1} \cdot S=0$. A set is exhaustible if it is the union of a countable collection of nowhere dense sets. A set is residual if it is the complement (with respect to $X$ ) of an exhaustible set. A set is inexhaustible if it is not exhaustible. The point $x$ is said to be a point of exhaustible f-approach if there is a $y$ in $f(x)$, an $M(y)$, and an $N(x)$ such that $\left[f^{-1}(M(y))\right] \cdot N(x)$ is exhaustible.

Presented to the Society, April 26, 1952; received by the editors March 7, 1952.

1 New properties of all real functions, Trans. Amer. Math. Soc. vol. 24 (1922) pp. 113-128. Also Arbitrary point transformations, Duke Math. J. vol. 11 (1944) pp. 671685. The definition of $f$-approach and $\lambda$ approach that we use here are essentially those introduced by Blumberg. 
If $x$ is not a point of exhaustible $f$-approach, then it is said to be a point of inexhaustible $f$-approach.

LEMma. The points of exhaustible f-approach form an exhaustible set.

Proof. Let $A$ be the set of points of exhaustible $f$-approach and let $x$ be an element of $A$. Then there is a $y$ in $f(x)$, an $M(y)$, and an $N(x)$ such that $\left[f^{-1}(M(y))\right] \cdot N(x)$ is exhaustible. Select $M^{k}(y) \subseteq M(y)$ and $N^{p}(x) \subseteq N(x)$. Let $A_{k, p}$ be the subset of $X$ given by: $A_{k, p}$ $=\left[f^{-1}\left(M^{k}(y)\right)\right] \cdot\left[N^{p}(x)\right]$. Then $x \in A_{k, p}$ and $A_{k, p}$ is exhaustible. Associate with each $x$ in $A$ such a pair of integers $k(x), p(x))$ and let $U$ be the union of the sets $A_{k(x), p(x)}$ thus defined. Then $A \subseteq U$. Since $U$ is exhaustible, so is $A$.

Definition. The point $x$ is said to be a point of concentrated inexhaustible $f$-approach if for each $y$ in $f(x)$ and each $M(y)$ there is an $N(x)$ such that for every $N_{\alpha} \subseteq N(x)$, the set $\left[f^{-1}(M(y))\right] \cdot N_{\alpha}$ is inexhaustible.

THEOREM. The points of concentrated inexhaustible f-approach form a residual set.

Proof. The proof will be based on the following lemma. In this way we illustrate a method of procedure which might also have been used in the proof of the preceding lemma.

Lemma. Let $\lambda$ be a set property, defined on all of $\mathfrak{R}$. The point $x$ is said to be a point of $\lambda$ approach if each $N(x)$ has the property $\lambda$. The point $x$ is said to be a point of concentrated $\lambda$ approach if there is an $N(x)$ such that any $N_{\alpha} \subseteq N(x)$ has the property $\lambda$. Then the set of points which are of $\lambda$ approach but are not of concentrated $\lambda$ approach is nowhere dense.

The proof is immediate.

We proceed with the proof of the theorem. Let $\lambda_{k}$ be the property of $N$ that $\left[f^{-1}\left(M^{k}\right)\right] \cdot N$ is inexhaustible. Let $B$ be the set of points of inexhaustible, but not concentrated inexhaustible, $f$-approach. Let $x$ be an element of $B$. For any $k$ such that $f(x) \cdot M^{k} \neq 0, x$ is a point of $\lambda_{k}$ approach (since $x$ is of inexhaustible $f$-approach). On the other hand (since $x$ is not a point of concentrated inexhaustible $f$-approach), there is some integer, $p$, such that: (i) $f(x) \cdot M^{p} \neq 0$, and (ii) $x$ is not a point of concentrated $\lambda_{p}$ approach. Associate with each $x \in B$ such a $p(x)$ and let $B_{m}$ be the union of all $x \in B$ thus associated with the integer $m$. Each $x \in B_{m}$ is then $\lambda_{m}$ approached, but not concentrated $\lambda_{m}$ approached. By the lemma, $B_{m}$ is nowhere dense, and, by construction, $B=\sum_{m} B_{m}$. Hence $B$ is exhaustible. Since the set of points of 
concentrated inexhaustible $f$-approach is $X-(A+B)$, it is residual.

In order to prove the corollaries that follow regarding continuity, we shall assume for the remainder of this paper that, in addition to the assumptions already made regarding $(X, \mathfrak{N}),(Y, \mathfrak{M})$, and $f$, the following conditions are also satisfied: (1) $f$ is single point valued; i.e., for each $x, f(x)$ is a single element of $Y$. (2) For any $M_{1}(y)$ and $M_{2}(y)$ there is an $M_{3}(y) \subseteq M_{1}(y) \cdot M_{2}(y)$. (3) $X$ is a Hausdorff space having the collection $\mathfrak{N}$ as a neighborhood system; i.e. each $N(x)$ is a neighborhood of $x$ in the Hausdorff sense. (4) Each $N$ is an inexhaustible set.

We also make some further definitions. If $S \subseteq X$, then the closure of $S$ is denoted by c $S$. A set of separated points is a set, $S$, such that for each $x$ in $S$ there is an $N(x)$ such that $\mathrm{c}[N(x)] \cdot(S-\{x\})=0$. The function $f_{S}$ is defined by $f_{S}(x)=f(x)$ for $x \in S$ and is undefined for $x \notin S$. We say that $f_{S}$ is continuous at $x \in S$ if, for each $M(f(x))$, there is an $N(x)$ such that, for every $\xi \in N(x) \cdot S, f(\xi) \in M(f(x))$. If $f_{S}$ is continuous at each $x \in S$, then $f_{S}$ is said to be continuous. The point $x \in S$ is said to be a point of concentrated inexhaustible $f_{S}$-approach if, for each $M(f(x))$, there is an $N(x)$ such that, for every $N_{\alpha} \subseteq N(x)$, $\left[f^{-1}(M(f(x)))\right] \cdot N_{\alpha} \cdot S$ is inexhaustible. Finally let $\Im$ denote the class of subsets of $X$ defined as follows: $S$ belongs to $\Im$ if and only if: (i) for each $N, N \cdot S$ is inexhaustible (i.e., $S$ is of the second Baire category at each point, and (ii) each $x \in S$ is of concentrated inexhaustible $f_{S}$-approach.

CoRollary 1. The class $\Im$ is not empty (for by condition (4) the residual set of the theorem belongs to $\Im$ ).

CoRollary 2. Let $I_{1}$ belong to $\Im$ and let $T$ be an arbitrary set of separated points belonging to $I_{1}$. Then there is an $I_{2}$ in $\Im$ such that $T \subseteq I_{2} \subseteq I_{1}$ and $f_{I_{2}}$ is continuous at each $x \in T$.

Proof. First suppose that $T$ consists of a single point, $\xi$. If $T$ is an $N(\xi)$, the proof is complete with $I_{2}=I_{1}$. If $T$ is not any $N(\xi)$, then it follows from condition (3) that there is a sequence of properly decreasing sets $N^{k_{1}}(\xi), N^{k_{2}}(\xi), \cdots, N^{k_{n}}(\xi), \cdots$ such that, for each $n, N^{k_{n}}-N^{k_{n}+1}$ contains an $N$ and, for each $N(\xi)$, there is an integer $p$ with $N^{k_{p}}(\xi) \subseteq N(\xi)$. Say $f(\xi)=\eta$. If $\eta$ is in no $M$, the proof is complete with $I_{2}=I_{1}$. If there is an $M(\eta)$, then by condition (2) we can take a sequence of nonincreasing sets: $M^{\gamma_{1}}(\eta), M^{\gamma_{2}}(\eta), \cdots$, $M^{\gamma_{n}}(\eta), \cdots$ (possibly terminating), such that for any $M(\eta)$ there is an integer $m$ with $M^{\gamma m} \subseteq M(\eta)$.

Now there is an $N^{k_{p}}(\xi)$ such that, for any $N \subseteq N^{k_{p}}(\xi),\left[f^{-1}\left(M^{\gamma_{1}}\right)\right]$ $\cdot N \cdot I_{1}$ is inexhaustible. Let $S_{1}$ be the set of all $x$ such that $f(x) \in M^{\gamma_{1}}$ 
and let $K_{1}=I_{1}-\left(\mathrm{c} N^{k_{p}}-S_{1} \cdot \mathrm{c} N^{k_{p}}\right) \cdot I_{1}$. Then $K_{1}$ belongs to $\Im$. If there is no $M^{\gamma_{2}}(\eta)$, we may stop. If there is one, then there is an $N^{k_{q}}(\xi)$ such that $q>p$ and, for any $N \subseteq N^{k_{q}}(\xi),\left[f^{-1}\left(M^{\gamma^{2}}\right)\right] \cdot N \cdot K_{1}$ is inexhaustible, since $M^{\gamma_{2}} \subseteq M^{\gamma_{1}}$. Take $S_{2}$ to be the set of all $x$ such that $f(x) \in M^{\gamma^{2}}$ and let $K_{2}=K_{1}-\left(\mathrm{c} N^{k_{q}}-S_{2} \cdot \mathrm{c} N^{k_{q}}\right) \cdot K_{1}$. Then $K_{2}$ belongs to $\Im$. Constructing, in this manner, a sequence $\left\{K_{n}\right\}$, we see that the set $I_{2}=\prod_{n} K_{n}$ has the properties stated.

If $T$ is an arbitrary set of separated points, we take, for each $x$ in $T$, a neighborhood $N_{x}(x)$ whose closure is disjoint from $T-\{x\}$ and carry out the same construction, starting the sequence of descending neighborhoods, $\left\{N_{x}^{k_{n}}\right\}$, about each $x$ of $T$, with the neighborhood $N_{x}(x)$.

CoROllary 3. Let $I_{1}$ belong to $\Im$ and let $F$ be any set consisting of $a$ finite number of points in $I_{1}$. Then there exists an everywhere dense set, $D$, with $F \subseteq D \subseteq I_{1}$, such that $f_{D}$ is continuous.

Proof. Select $\xi_{1}$ arbitrarily from $N^{1} \cdot I_{1}$. Apply Corollary 2 (replacing $T$ there by $F+\left\{\xi_{1}\right\}$ ), obtaining the set $I_{2}$. Proceed in this manner; viz., at the $j$ th step take $\xi_{j}$ arbitrarily from $N^{j} \cdot I_{j}$ and apply Corollary 2 (replacing $I_{1}$ there by $I_{j}, T$ by $\left(F+\left\{\xi_{1}\right\}+\left\{\xi_{2}\right\}+\cdots\right.$ $\left.+\left\{\xi_{j}\right\}\right)$, and $I_{2}$ by $\left.I_{j+1}\right)$. The set $D=\left(F+\left\{\xi_{1}\right\}+\left\{\xi_{2}\right\}+\cdots+\left\{\xi_{n}\right\}\right.$ $+\cdots)$ then has the properties stated.

Definition. We say that the Hausdorff space $(X, \mathfrak{N})$ has the property $\mathrm{P}$ if, for each $N$, there is an $N_{1}$ such that c $N_{1} \subseteq N$.

We remark that the property $P$ does not imply that, for a given $N$ and a given $x \in N$, there exists a $\mathrm{c}\left[N_{1}(x)\right] \subseteq N$. Indeed this last condition is equivalent to regularity of the Hausdorff space. A regular Hausdorff space has the property $\mathrm{P}$ but there exist Hausdorff spaces having the property $\mathrm{P}$ which are irregular at each point. ${ }^{2}$

Corollary 4. Let the Hausdorff space $(X, \mathfrak{N})$ have the property $\mathrm{P}$. Let $I_{1}$ belong to $\Im$ and let $T$ be any set of separated points in $I_{1}$. Then there exists an everywhere dense set, $D$, with $T \subseteq D \subseteq I_{1}$, such that $f_{D}$ is continuous.

Proof. The proof given for Corollary 3 is suitable with the modification that, in selecting a $\xi_{j}$ from $N^{j}$, we require that if $N^{j}$ contains any points of $T$, then $\xi_{j}$ shall be selected from among these. If no point of $T$ is in $N^{j}$, then we find a $c N_{j} \subseteq N^{j}$ and select $\xi_{j}$ from $N_{j}$. In this case $\left(T+\left\{\xi_{1}\right\}+\cdots+\left\{\xi_{j}\right\}\right)$ remains a set of separated points and the construction can proceed.

2 Examples of such spaces, as well as many helpful suggestions, were given to the writers by Professor H. P. Thielman. 
REMARK. The set $D$ in the last two corollaries is denumerable. That this result cannot be improved (assuming the continuum hypothesis) follows from the construction of Sierpinski and Zygmund ${ }^{3}$ of a real function of a real variable, $f(x)$, such that, for any set, $I$, having the cardinality of the continuum, $f_{I}$ is not continuous.

All the results of the present paper are of course applicable to an arbitrary operator on a separable Banach space. Somewhat related results are true for linear operators on an arbitrary Banach space ${ }^{4}$ (the linearity of the operator making up for, in a sense, the lack of a denumerable basis).

Iowa State College of Agriculture and Mechanical Arts

Sur une fonction qui est discontinue sur tout ensemble de puissance du continu, Fund. Math. vol. 4 (1923) p. 316. Also W. Sierpinski, Hypothese du continu, Warsaw, 1934, p. 118. (Monografje Mathematyczne No. 4.)

4. D. Block, Linear transformations on or onto a Banach space, Proceedings of the American Mathematical Society vol. 3 (1952) p. 126. 